\title{
Asociación de sialometría, fosfato y calcio en saliva total bajo estímulo y en líquido crevicular gingival con caries dental en escolares
}

\author{
Nesby Velásquez¹, Luis Pérez-Ybarra², Carmen J. Urdaneta1', Mariela Pérez- \\ Domínguez ${ }^{1,3}$ \\ 1 Unidad de Investigaciones Morfopatológicas, Facultad de Odontología, Universidad de \\ Carabobo, Valencia, Venezuela \\ ${ }^{2}$ Departamento de Ciencias Básicas, Escuela de Bioanálisis, sede Aragua, Facultad de Ciencias \\ de la Salud, Universidad de Carabobo, Maracay, Venezuela \\ ${ }^{3}$ Departamento de Ciencias Morfofuncionales, Facultad de Odontología, Universidad de \\ Carabobo, Valencia, Venezuela
}

Introducción. La capacidad de la saliva para la remineralización contribuye a mantener la integridad fisicoquímica de la estructura mineral de los dientes, lo cual los protege de la aparición y evolución de la caries dentaria.

Objetivo. Establecer la relación de la sialometría, la capacidad amortiguadora, la concentración de calcio y fosfato en la saliva total estimulada y en el líquido crevicular gingival, con la gravedad y la actividad de las caries en escolares.

Materiales y métodos. Se seleccionaron 36 escolares de 6 años, 18 con caries (International Caries Detection and Assessment System, ICDAS, código 2 o mayor) y 18 sin caries (ICDAS, código 0). En la dentición primaria, se evaluaron la gravedad y la actividad de la caries dentaria en la superficie de oclusión de los molares y en la vestibular de los dientes anteriores, y se codificaron mediante el ICDAS-II.

Recibido: 07/09/17

Aceptado: 22/08/18

Publicado: 27/08/18

Citación:

Velásquez N, Pérez-Ybarra L, Urdaneta C, PérezDomínguez M. Asociación de sialometría, fosfato y calcio en saliva total bajo estímulo y en líquido crevicular gingival con caries dental en escolares. Biomédica. 2018;39:157-69

https://doi.org/10.7705/biomedica.v39i1.4069

\section{Correspondencia:}

Mariela Pérez-Domínguez, Facultad de Odontología, Universidad de Carabobo, pabellón 11, Ciudad Universitaria Bárbula, Estado Carabobo, Valencia, Venezuela

Teléfono: (58 424) 4956321

marieldp1@gmail.com

\section{Contribución de los autores:}

Mariela Pérez-Domínguez: diseño de la investigación, procesamiento de las muestras biológicas, recopilación de la información bibliográfica, supervisión y revisión de todas las etapas de la investigación

Nesby Velásquez: recolección de las muestras biológicas y evaluación clínico odontológica de los escolares, procesamiento de las muestras biológicas Luis Pérez-Ybarra: diseño de la investigación, análisis estadístico, recopilación de la información bibliográfica, supervisión y revisión de todas las etapas de la investigación

Carmen J. Urdaneta: procesamiento de las muestras biológicas

Todos los autores participaron en la redacción del artículo.

Financiación:

El estudio se financió con recursos propios.

Resultados. Se evidenció una mayor gravedad de las caries en la superficie de oclusión que en la vestibular. Las concentraciones de calcio en la saliva y de fosfato en el líquido crevicular gingival de los dientes sanos, fueron mayores en el grupo con un código ICDAS de 20 mayor. La concentración de calcio en el líquido crevicular gingival fue mayor en el grupo con código ICDAS 0 que en los dientes cariados del grupo con código ICDAS de 2 o mayor. Se encontró una asociación estadísticamente significativa entre la frecuencia de caries activas y la concentración de fosfato en el líquido crevicular gingival de los dientes afectados, como también entre la gravedad de las caries y la capacidad amortiguadora, por una parte, y la concentración de fosfato en el líquido crevicular gingival de los dientes afectados, por la otra. Conclusión. Se evidenció una asociación entre la caries dental, la capacidad amortiguadora y la química bucal del calcio y el fosfato.

Palabras clave: caries dental; saliva; líquido del surco gingival; niño.

Sialometry and concentration of phosphate and calcium in stimulated whole saliva and gingival crevicular fluid and its association with dental caries in schoolchildren

Introduction: The remineralizing properties of saliva contribute to maintain the physical and chemical integrity of the mineral structure of teeth, which protects it from the installation and evolution of dental caries.

Objective: To relate sialometry, buffering capacity, calcium and phosphate concentration in whole stimulated saliva, and in gingival crevicular fluid with school children caries severity and activity.

Materials and methods: We selected 36 schoolchildren aged 6 years: 18 with caries (International Caries Detection and Assessment System, ICDAS $>1$ group) and 18 without caries (ICDAS=0 group). The severity and activity of dental caries were diagnosed in the primary dentition: in the occlusal surface of molars and in the vestibular of the anterior teeth by ICDAS-II. Results: Caries in occlusal surface were more severe than in vestibular surface. The concentration of calcium in saliva and phosphate in healthy teeth gingival crevicular fluid were higher in the ICDAS>1 group. The concentration of calcium in gingival crevicular fluid was higher in the ICDAS=0 group than in the decayed teeth of the ICDAS $>1$ group. We found a statistically significant association between the frequency of active caries andthe concentration of phosphate in gingival crevicular fluid of teeth with caries, as well as between the severity of caries with buffering capacity and the concentration of phosphate in the gingival crevicular fluid of teeth with caries.

Conclusion: We found an association between dental caries with buffering capacity and buccal calcium and phosphate.

Key words: Dental caries; saliva; gingival crevicular fluid; child. 
La caries dental es un problema de salud pública con un alto grado de morbilidad y elevada prevalencia (1), que afecta a casi el $99 \%$ de la población mundial. Se ha reportado que esta enfermedad afecta entre el 60 y el $90 \%$ de los niños en edad escolar (2), y su distribución y gravedad varían en diferentes partes del mundo y dentro de una misma región o país. En las Américas, su prevalencia oscila entre el 45 y el $85 \%$ (3-7). En el último reporte nacional, en Venezuela, se registró una prevalencia del $55 \%$ en niños entre los 5 y los 12 años de edad, así como disminución de sus índices (8). En otros estudios en niños, en los que se utilizó el International Caries Detection and Assessment System II (ICDAS II), se detectó que la caries leve o de etapa temprana era la más frecuente $(9,10)$.

El desarrollo de la caries dentaria se inicia cuando se rompe el equilibrio dinámico entre remineralización y desmineralización, lo que afecta la integridad fisicoquímica de la apatita del esmalte debido a la liberación e incorporación de iones como el calcio y el fosfato $(11,12)$. Este proceso se observa más frecuentemente en las superficies de oclusión (puntos, fosas y fisuras profundas) que en las superficies lisas (13-15), por las condiciones de los microambientes biológicos y la exposición sistemática a los ácidos orgánicos procedentes del metabolismo de la biopelícula dentobacteriana. Las condiciones salivales que no neutralizan los ácidos orgánicos pueden favorecer la enfermedad.

En algunos estudios se ha registrado cómo la tasa de flujo salival, el pH, la capacidad amortiguadora y la concentración de calcio en la saliva, presentaban valores inferiores en escolares con caries dentaria (16-18). No obstante, no está claro si esto se asociaba con la actividad y la gravedad de la enfermedad, o si los iones presentes en la saliva y en el líquido crevicular gingival, o líquido del surco gingival, presentaban diferencias en los dientes cariados.

Por lo tanto, en el presente estudio se evaluaron los parámetros de sialometría (velocidad de flujo salival), concentración de las moléculas responsables de la capacidad amortiguadora de la saliva, $\mathrm{pH}, \mathrm{y}$ concentración de calcio y fosfato, tanto en la saliva como en el líquido crevicular gingival, en niños en edad escolar atendidos en el área clínica del posgrado de Odontopediatría de la Universidad de Carabobo, con el fin de verificar si tales parámetros estaban asociados con la gravedad y la actividad de la caries dentaria en la dentición primaria.

\section{Materiales y métodos}

Se hizo un estudio de tipo descriptivo y correlacional con diseño transversal. La población estaba conformada por 275 escolares de ambos sexos atendidos entre julio y octubre de 2015 en al área clínica del posgrado de Odontopediatría de la Universidad de Carabobo.

Se seleccionó una muestra de 36 escolares de 6 años de edad y se los distribuyó en dos grupos de 18 niños cada uno: el grupo ICDAS>1 (con caries) y el grupo ICDAS $=0$ (sin caries), cada uno conformado por nueve niños y nueve niñas. La selección de los sujetos fue intencional según los criterios de inclusión del estudio y se mantuvo la misma cantidad de individuos en cada grupo, determinada por el número mínimo de niños del mismo sexo seleccionados durante el periodo de estudio, es decir, nueve niños de cada sexo.

Se excluyeron los escolares con tratamientos odontológicos de ortopedia u ortodoncia, tratamientos farmacológicos con medicamentos 
agonistas o antagonistas colinérgicos y adrenérgicos, enfermedades infecciosas o traumatismos que afectaran las glándulas salivales mayores o enfermedades sistémicas.

Todos los escolares que participaron en el estudio accedieron a hacerlo de forma voluntaria y sus padres o representantes firmaron el consentimiento informado en cumplimiento de las Normas de la Comisión de Bioética y Bioseguridad del Fondo Nacional de Ciencia, Tecnología e Innovación (FONACIT), y de la evaluación del proyecto No. SPBBOP-037-2016 por parte de la Comisión de Bioética de la Facultad de Odontología de la Universidad de Carabobo.

\section{Toma de muestras y análisis de laboratorio}

La saliva total se recolectó bajo estímulo entre las 8:00 y las 11:00 a.m. Los escolares que participaron no consumieron alimentos ni bebidas y no se cepillaron los dientes durante los 90 minutos previos a la prueba. Se les instruyó para enjuagarse vigorosamente la boca con agua, tragar la primera secreción de saliva y, enseguida, masticar un gramo de cera (Parafilm M, Pechiney, Chicago, Illinois, USA) durante seis minutos, escupiendo cada 30 segundos.

Las muestras de líquido del surco gingival se obtuvieron de tres piezas de la dentición primaria de los escolares del grupo ICDAS $=0 \mathrm{y}$, de seis piezas de la dentición primaria de los del grupo ICDAS $>1$ : tres con caries y tres sin caries. En el grupo ICDAS $=0$ se evaluaron los dos dientes caninos y un segundo molar superior, y en el ICDAS $>1$, los dos primeros molares superiores y un segundo molar superior con caries, así como dos dientes caninos o incisivos inferiores y el primer o segundo molar superior sin caries.

Las muestras se recolectaron con puntas estériles de papel de endodoncia No. 25, que se colocaron en el surco gingival de cada diente (medial, distal, vestibular, lingual o palatino), tres por zona, durante 10 segundos. Las puntas contaminadas con sangre se descartaron (19).

Las muestras de saliva total bajo estímulo se sometieron a pruebas para determinar su sialometría, el $\mathrm{pH}$, la capacidad amortiguadora de la saliva y las concentraciones de calcio y fosfato inorgánico, en tanto que en las muestras de líquido del surco gingival, se determinaron la concentración de calcio y la de fosfato inorgánico.

La determinación de la sialometría y del pH se hizo inmediatamente después de recolectar las muestras de saliva. Se registró el volumen total de saliva obtenido bajo estímulo en seis minutos y la tasa de flujo, es decir, la cantidad de saliva por minuto ( $\mathrm{ml} /$ minuto).

El pH salival se obtuvo con un medidor de $\mathrm{pH}$ portátil (Hanna $\left.{ }^{\mathrm{TM}}\right)$ y la capacidad amortiguadora de la saliva $(\mathrm{mmol}-\mathrm{HCl} / \mathrm{ml})$ se estableció mediante la titulación de $1 \mathrm{ml}$ de saliva con $\mathrm{HCl} 0,1 \mathrm{~N}$ y una gota del indicador colorimétrico de $\mathrm{pH}$ naranja de metilo $(\mathrm{pH} 3,1$ a 4,4), procedimiento que fue repetido tres veces y se expresó como milimoles de ácido añadidos a $1 \mathrm{ml}$ de saliva (20).

Para determinar la concentración del calcio $(\mathrm{mM})$ y el fosfato inorgánico $(\mathrm{mM})$ en saliva bajo estímulo y en líquido del surco gingival, se emplearon, respectivamente, los métodos colorimétricos $\mathrm{Ca}$-Color AA y fosfatemia UV AA de Wiener Lab., según las indicaciones del fabricante. Las pruebas se hicieron por triplicado y las concentraciones se estimaron promediando las tres mediciones. 


\section{Diagnóstico de caries dental}

Se hizo un examen clínico odontológico en los escolares para establecer el diagnóstico de caries dental, según los criterios del ICDAS II (21), modificados por Zambrano, et al. (22).

Dada su conocida sensibilidad, solo se evaluaron las superficies de oclusión de los dientes molares primarios y las superficies vestibulares de los dientes anteriores primarios (de canino a canino).

El diagnóstico de la seriedad de la caries dental se basó en los criterios establecidos para el estadio de caries temprana, establecida o grave. La actividad de la caries también se determinó clínicamente según el código ICDAS II $(21,23)$.

El examen clínico dental se efectuó en el área clínica del posgrado de Odontopediatría de la Facultad de Odontología de la Universidad de Carabobo, utilizando para ello un espejo bucal plano, la sonda periodontal sugerida por la Organización Mundial de la Salud (OMS) y calibrada en 11,5, $8,5,5,5$ y $3,5 \mathrm{~mm}$, y una pinza algodonera.

La presencia de caries dentarias activas e inactivas se expresó en términos de porcentaje de caries por niño, y se calculó para las superficies de oclusión y vestibular como la razón entre el número de dientes cariados y el número de dientes del niño para cada superficie considerada.

\section{Análisis estadístico}

Para garantizar el cabal cumplimiento de las directrices del ICDAS II por parte del examinador clínico, se calculó el coeficiente kappa de concordancia de Cohen entre los resultados obtenidos por este y los de un experto.

Se calcularon los parámetros estadísticos descriptivos de media aritmética y desviación estándar para las variables de sialometría, $\mathrm{pH}$, capacidad amortiguadora, calcio y fosfato en saliva y en líquido del surco gingival clasificadas por grupo, y a estas se les aplicó la prueba de medias t de Student. Para las variables de concentración de calcio y fosfato en saliva y en líquido del surco gingival, se calcularon los coeficientes de correlación de Pearson dentro de cada grupo.

En el grupo ICDAS>1 se usó la prueba de Wilcoxon-Mann-Whitney para las variables de presencia, actividad y gravedad de las caries, clasificadas según la superficie del diente analizada (de oclusión o vestibular). En este grupo también se calcularon los coeficientes de correlación de rangos de Spearman entre las variables asociadas con la actividad y la gravedad de las caries, y las asociadas con la concentración de calcio y fosfato en saliva y en líquido del surco gingival.

Los niveles de significación se fijaron en 5 y $10 \%$, por lo cual una diferencia o asociación de $5 \%$ se consideró estadísticamente significativa siempre que $p$ fuera de 0,05 o menor $y$, una de $10 \%$, si el valor de $p$ era mayor de 0,05 y menor o igual a 0,1 . Los datos se procesaron utilizando los programas estadísticos Statistix 9.0 ${ }^{\mathrm{TM}}$ y Minitab 16.0 ${ }^{\mathrm{TM}}$, ambos bajo ambiente Windows.

\section{Resultados}

El examinador clínico fue calibrado para la detección de caries dentaria, según los criterios del ICDAS II, por Olga Zambrano de la Universidad del Zulia, Venezuela (patrón nacional del país para la calibración de examinadores según el ICDAS II). 
El índice kappa de concordancia entre examinadores fue de 0,86 y el coeficiente para el examinador consigo mismo fue de 0,75 , lo cual representó una excelente concordancia entre ambos examinadores (24) e indicó que los resultados se ajustaban a los criterios del ICDAS II.

\section{Características de las piezas dentales analizadas}

Grupo ICDAS=0. En general, se evaluaron 144 superficies de oclusión de dientes molares primarios y 180 superficies vestibulares de dientes anteriores primarios en los 18 niños seleccionados. De estos dientes faltaban 36 debido al proceso de recambio dental.

Grupo ICDAS>1. En los 18 niños seleccionados se evaluaron 141 superficies de oclusión de dientes molares primarios, de las cuales $63(44,68$ $\%)$ resultaron sanas. Las restantes $78(55,32 \%)$ presentaban caries y, de ellas, $19(13,48 \%)$ presentaban caries en estadio temprano, $27(19,15 \%)$, caries establecidas, y 32 (22,70\%), caries graves. Asimismo, 77 (54,61\%) caries estaban activas y, además, se observó la ausencia de tres molares primarios.

Por otra parte, se evaluaron 182 superficies vestibulares de dientes anteriores primarios, de las cuales $150(82,42 \%)$ resultaron sanas, en tanto que las restantes $32(17,58 \%)$ presentaban caries; de ellas, $7(3,85 \%)$ presentaban caries en estado temprano, $18(9,89 \%)$, caries establecidas, y $7(3,85 \%)$, caries graves. Asimismo, 31 (17,03\%) caries estaban activas y, además, se observó la falta de 32 piezas primarias debido al proceso de recambio dental.

\section{Parámetros de sialometría, capacidad amortiguadora, $\mathrm{pH}$, calcio y fosfato en saliva total bajo estímulo y en líquido crevicular gingival}

La prueba t de Student indicó que había diferencias estadísticamente significativas en el grupo ICDAS $=0$ para el calcio en saliva $(p=0,0028)$, el calcio en líquido crevicular gingival de los dientes con caries del grupo ICDAS $>1(p<0,0001)$ y el fosfato en líquido crevicular gingival de los dientes sanos del grupo ICDAS $>1$ ( $p<0,0001)$ (cuadro 1). La concentración de calcio en saliva y de fosfato en líquido crevicular de dientes sin caries fue mayor en el grupo ICDAS>1, en tanto que la concentración de Ca en líquido crevicular de dientes cariados fue mayor en el grupo ICDAS $=0$. No se observaron diferencias estadísticamente significativas en el resto de las variables.

Cuadro 1. Parámetros de sialometría, $\mathrm{pH}$, capacidad amortiguadora, calcio y fosfato en saliva total bajo estímulo y en líquido crevicular gingival clasificados por grupo

\begin{tabular}{lccc}
\hline \multicolumn{1}{c}{ Variable } & ICDAS>1 & ICDAS=0 & p \\
\hline Volumen total de saliva (DE) (ml) & $8,97(1,43)$ & $8,57(1,41)$ & 0,4042 \\
Flujo salival (DE) (ml/minuto) & $1,50(0,24)$ & $1,43(0,24)$ & 0,4042 \\
pH (DE) & $8,03(0,36)$ & $8,07(0,34)$ & 0,7756 \\
Capacidad amortiguadora (DE) (mmol-HCl/ml-saliva) & $8,50(1,99)$ & $8,19(1,88)$ & 0,6384 \\
Calcio en saliva (DE) (mM) & $2,91(0,97)$ & $1,81(1,07)$ & $0,0028^{*}$ \\
Fosfato en saliva (DE) (mM) & $33,62(6,15)$ & $32,65(9,24)$ & 0,6042 \\
Calcio en LCG para dientes sanos del grupo ICDAS>1 (DE) (mM) & $1,87(1,05)$ & $1,58(1,12)$ & 0,4166 \\
Calcio en LCG para dientes con caries del grupo ICDAS>1 (DE) (mM) & $0,44(0,46)$ & $1,58(1,12)$ & $<0,0001^{*}$ \\
Fosfato en LCG para dientes sanos del grupo ICDAS>1 (DE) (mM) & $4,50(1,45)$ & $0,81(2,39)$ & $<0,0001^{*}$ \\
Fosfato en LCG para dientes con caries del grupo ICDAS>1 (DE) (mM) & $0,73(0,19)$ & $0,81(2,39)$ & 0,8890 \\
\hline
\end{tabular}

DE: desviación estándar; LCG: líquido crevicular gingival

* Diferencia estadísticamente significativa al $5 \%$ 
Por otra parte, la prueba t de Student no evidenció diferencias estadísticamente significativas $(p>0,10)$ dentro de cada grupo de estudio para ninguna de las variables analizadas según el sexo de los niños (no se presentan los resultados).

El coeficiente de correlación de Pearson entre las concentraciones de calcio y de fosfato en la saliva y en el líquido del fondo gingival, no reveló una asociación estadísticamente significativa $(p>0,10)$ en ninguno de los dos grupos bajo estudio (no se presentan los resultados).

Se observaron diferencias estadísticamente significativas según la prueba de Wilcoxon-Mann-Whitney para las variables de frecuencia de las caries $(p=0,0001)$, frecuencia de las caries activas $(p=0,0001)$ y gravedad de las caries $(p=0,0003)$. Todas estas variables presentaron valores mayores en la superficie de oclusión, lo cual indicaba que en la muestra analizada la presencia y la gravedad de las caries era mayor en los dientes molares que en los anteriores (cuadro 2).

Cuadro 2. Frecuencia, actividad y gravedad de las caries en las superficies analizadas en el grupo ICDAS>1

\begin{tabular}{lccc}
\hline \multicolumn{1}{c}{ Variable } & De oclusión & Vestibular & p \\
\hline Dientes con caries (DE) (\%) & $56,05(28,72)$ & $18,05(18,77)$ & $0,0001^{*}$ \\
Dientes con caries activas (DE) (\%) & $55,95(27,16)$ & $17,49(19,16)$ & $0,0001^{*}$ \\
Gravedad de las caries (DE) (\%) & $2,72(1,16)$ & $1,44(0,56)$ & $0,0003^{*}$ \\
\hline
\end{tabular}

DE: desviación estándar

* Diferencia estadísticamente significativa al $5 \%$

Cuadro 3. Coeficientes de correlación de Spearman entre los parámetros de sialometría, pH, capacidad amortiguadora, calcio y fosfato en saliva total bajo estímulo y en líquido crevicular gingival, y la actividad y la gravedad de las caries en el grupo ICDAS>1

\begin{tabular}{|c|c|c|c|c|}
\hline \multirow{2}{*}{ Variables } & \multicolumn{2}{|c|}{ Caries activas (\%) } & \multicolumn{2}{|c|}{ Gravedad de la caries } \\
\hline & De oclusión & Vestibular & De oclusión & Vestibular \\
\hline \multirow[t]{2}{*}{ Saliva } & $r=0,1507$ & $r=0,1023$ & $r=-0,0695$ & $r=-0,0230$ \\
\hline & $p=0,5467$ & $p=0,6804$ & $p=0,7859$ & $p=0,9279$ \\
\hline \multirow[t]{2}{*}{ Flujo salival } & $r=0,1507$ & $r=0,1023$ & $r=-0,0695$ & $r=-0,0230$ \\
\hline & $p=0,5467$ & $p=0,6804$ & $p=0,7859$ & $p=0,9279$ \\
\hline \multirow[t]{2}{*}{$\mathrm{pH}$} & $r=-0,2169$ & $r=-0,0618$ & $r=-0,3861$ & $r=-0,0740$ \\
\hline & $p=0,3838$ & $p=0,8050$ & $p=0,1135$ & $p=0,7670$ \\
\hline \multirow[t]{2}{*}{ Capacidad amortiguadora } & $r=0,3870$ & $r=0,2761$ & $r=0,5245$ & $r=0,3381$ \\
\hline & $p=0,1115$ & $p=0,2637$ & $p=0,0270^{*}$ & $p=0,1681$ \\
\hline \multirow[t]{2}{*}{ Calcio en saliva } & $r=-0,0566$ & $r=0,0347$ & $r=-0,2754$ & $r=0,1482$ \\
\hline & $p=0,8242$ & $p=0,8888$ & $p=0,2674$ & $p=0,5522$ \\
\hline \multirow[t]{2}{*}{ Fosfato en saliva } & $r=0,2416$ & $r=-0,1121$ & $r=0,3988$ & $r=-0,0940$ \\
\hline & $p=0,3308$ & $p=0,6563$ & $p=0,1017$ & $p=0,7109$ \\
\hline \multirow[t]{2}{*}{ Calcio en dientes sanos } & $r=0,0838$ & $r=0,1494$ & $r=0,1563$ & $r=0,1232$ \\
\hline & $p=0,7357$ & $p=0,5467$ & $p=0,5302$ & $p=0,6208$ \\
\hline \multirow[t]{2}{*}{ Calcio en dientes con caries } & $r=0,0136$ & $r=-0,3136$ & $r=0,0104$ & $r=-0,3372$ \\
\hline & $p=0,9541$ & $p=0,2029$ & $p=0,9672$ & $p=0,1709$ \\
\hline \multirow[t]{2}{*}{ Fosfato en dientes sanos } & $r=-0,0944$ & $r=0,2022$ & $r=-0,1171$ & $r=0,2492$ \\
\hline & $p=0,7109$ & $p=0,4168$ & $p=0,6444$ & $p=0,3142$ \\
\hline \multirow[t]{2}{*}{ Fosfato en dientes con caries } & $r=0,4195$ & $r=-0,1210$ & $r=0,4363$ & $r=-0,0372$ \\
\hline & $p=0,0840^{* *}$ & $p=0,6325$ & $p=0,0702^{* *}$ & $p=0,8823$ \\
\hline
\end{tabular}

FS: flujo salival; CA: capacidad amortiguadora

* Asociación estadísticamente significativa al $5 \%$

${ }^{* *}$ Asociación estadísticamente significativa al $10 \%$ 
No se encontró una asociación estadísticamente significativa según el coeficiente de correlación de rangos de Spearman entre la gravedad y la actividad de la caries en la superficie vestibular y las variables de sialometría, $\mathrm{pH}$, capacidad amortiguadora, calcio y fosfato en saliva total bajo estímulo y en líquido crevicular.

En cuanto a la frecuencia de caries activas, se encontró una asociación estadísticamente significativa con la concentración de fosfato en el líquido crevicular de los dientes con caries $(r=0,4195 ; p=0,0840)$. Con respecto a la gravedad de las caries, se encontró una asociación estadísticamente significativa entre esta, la capacidad amortiguadora $(r=0,5245 ; p=0,0270)$ y la concentración de fosfato en el líquido crevicular de los dientes con caries $(r=0,4363 ; p=0,0702)$ (cuadro 3).

Todas las asociaciones encontradas fueron directamente proporcionales, es decir, cuanto mayores los valores de una variable, mayores también los de la variable asociada (figura 1). En esta figura se presentan los gráficos de dispersión y las líneas de tendencia de mínimos cuadrados para las asociaciones detectadas según el coeficiente de correlación de rangos de Spearman.
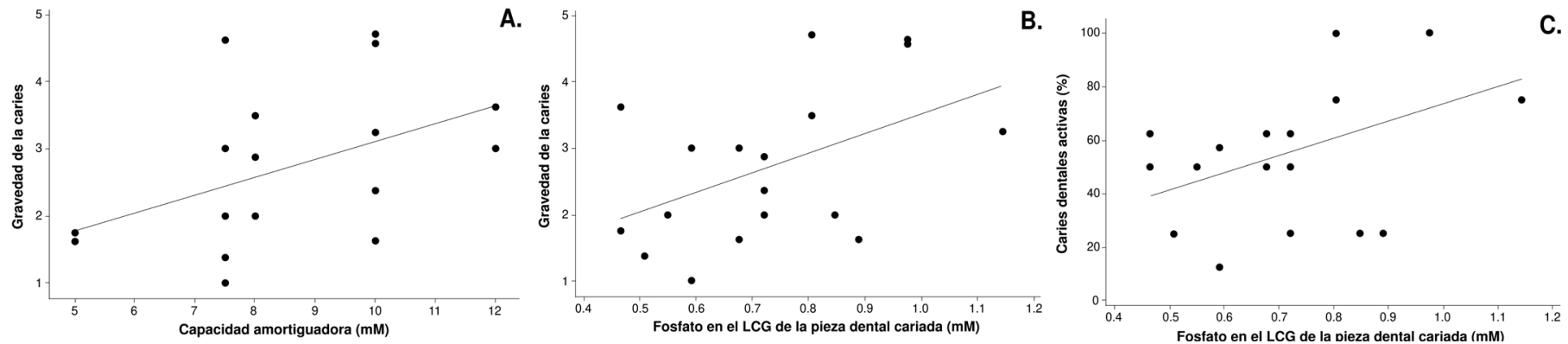

LCG: líquido crevicular gingival

Figura 1. Gráficos de dispersión de las asociaciones encontradas en los dientes molares primarios de escolares con caries dental. A. Capacidad amortiguadora y gravedad de las caries. B. Fosfato en líquido crevicular gingival en dientes cariados y gravedad de las caries. C. Fosfato en LCG en dientes cariados y caries activas

\section{Discusión}

En el presente trabajo se encontró una mayor frecuencia de caries dentaria en las superficies de oclusión que en las vestibulares, así como un mayor número de caries establecidas.

Díaz-Cárdenas, et al. (25), observaron hallazgos similares; reportaron que las superficies de oclusión, seguidas de las vestibulares, fueron las más afectadas por la enfermedad y que el estadio de las caries establecida fue el de mayor frecuencia. La vulnerabilidad de estas superficies se ha relacionado con características morfológicas como la convexidad, la concavidad y las fisuras, entre otras $(15,26,27)$, así como con la microbiota que las coloniza como resultado del elevado consumo de carbohidratos fermentables en la dieta de los escolares, y con la saliva y la influencia genética $(28,29)$.

Otro estudio en escolares reportó una mayor frecuencia de caries dentaria en el esmalte, aunque en estadio inicial; además, en la mayoría de las superficies examinadas con evidencia de caries, estas estaban activas, aunque con diversos grados de gravedad (30). Resultados similares fueron reportados por Piovesan, et al. (31), quienes observaron una mayor frecuencia de las lesiones de caries activas sin cavitaciones. Asimismo, 
Guedes, et al. (32), observaron que las lesiones por caries activas en superficies de oclusión sin cavitaciones presentaban mayor riesgo de progresión que aquellas con lesiones inactivas e, inclusive, un mayor riesgo de desarrollar nuevas lesiones.

El flujo salival se ha correlacionado con las enfermedades orales $(16,33)$. No obstante, en este estudio se evidenció que el volumen total de saliva bajo estímulo y el flujo salival no presentaron variaciones importantes según el grupo y el sexo, como tampoco se detectó una correlación con la frecuencia, la gravedad o la actividad de la caries dentaria. Los resultados obtenidos por Pandey, et al. (17), fueron similares; los autores demostraron que no había una correlación significativa entre la actividad de las caries y la tasa de flujo salival. Tampoco Maeda, et al. (34), Dogra, et al. (35), o Sullivan (36), encontraron una asociación estadísticamente significativa entre el flujo salival y la caries dentaria, aunque le atribuyeron alguna participación en su etiología y patogenia.

Sin embargo, las conclusiones de otras investigaciones no concuerdan con las de este trabajo. Sakeenabi, et al. (37), demostraron una alta correlación entre la frecuencia de caries y el flujo salival. Asimismo, Kaur, et al. (38), Prabhakar, et al. (39) y Fiyaz, et al. (40), demostraron que el flujo salival fue menor en los niños con caries dental activa que en aquellos sin caries. Por su parte, Animireddy, et al. (41), observaron que los niños con caries dentaria presentaban una disminución significativa en la tasa de flujo salival y, además, lo consideraron como indicador de la actividad de la caries.

En el presente estudio, los valores de $\mathrm{pH}$ en el grupo ICDAS>1 oscilaron entre 7,2 y 8,5 , en tanto que en el grupo ICDAS=0, entre 6,8 y 8,3. La propiedad amortiguadora de la saliva total con valores de $\mathrm{pH}$ de 5,1 y 8 se atribuye al hidrogenocarbonato de sodio, al dihidrogenofosfato de sodio y a una gran fracción de proteínas, entre las cuales, la alfa-amilasa parece tener una participación importante (42).

Sin embargo, si estos sistemas no logran compensar la producción de ácidos orgánicos generados por el metabolismo microbiano a partir de una dieta rica en carbohidratos fermentables, el pH disminuye y se favorece la desmineralización dentaria, lo que permite el desarrollo de la caries dentaria (17). Se ha demostrado, sin embargo, que los individuos con una higiene bucal deficiente, pero con un $\mathrm{pH}$ elevado, compensado por una eficiente capacidad amortiguadora, tienen menor tendencia a desarrollar caries dental (40).

En este estudio, la capacidad amortiguadora de la saliva y el $\mathrm{pH}$ presentaron promedios similares en los escolares con caries dentaria y en aquellos con dentadura sana, aunque se encontró una asociación significativa entre la capacidad amortiguadora, y la frecuencia y gravedad de las caries en los molares, si bien no hubo asociación con la actividad de la enfermedad. En concordancia con este estudio, Maeda, et al. (34), Dogra, et al. (35), Sakeenabi, et al (37) y Animireddy, et al. (41), demostraron una disminución significativa del $\mathrm{pH}$ y de la capacidad amortiguadora en pacientes con caries dentaria.

Por el contrario, Pandey, et al. (17), no detectaron una correlación significativa entre los valores de $\mathrm{pH}$ y la actividad de las caries, la edad y el sexo. Asimismo, Prabhakar, et al. (39), evidenciaron que el pH y la capacidad amortiguadora presentaban poca correlación con la actividad de las caries, resultados que pueden atribuirse a que el $\mathrm{pH}$ salival y la capacidad amortiguadora, evaluados por sí solos, no representan factores confiables para la predicción del riesgo de caries en la primera infancia (43), pero sí parecen tener un papel en su evolución. 
Las altas concentraciones de calcio y fosfato en la saliva favorecen el intercambio iónico durante el proceso de remineralización del esmalte, incrementan su resistencia y revierten lesiones que no presentan cavitaciones (38). En el presente trabajo se encontró que la concentración de calcio en la saliva total bajo estímulo tenía una media significativamente superior en los escolares con caries dentaria, aunque no hubo asociación con su actividad, en tanto que la concentración de fosfato fue similar en ambos grupos. Se encontró, además, que las concentraciones de calcio en el exudado gingival de piezas con caries eran inferiores a la media del grupo ICDAS=0, mientras que las de fósforo en el mismo líquido fueron superiores a la media del grupo ICDAS=0. También, se evidenció que no existía correlación entre las concentraciones de calcio y de fosfato salival en el líquido crevicular gingival de los grupos con caries y sin ellas.

En contraste con estos resultados, Kargül, et al. (44), registraron diferencias no significativas en las concentraciones de calcio y fosfato salival entre niños con caries y sin ellas. Kaur, et al. (38), reportaron diferencias no significativas en las concentraciones de calcio en saliva sin estímulo entre niños con caries y sin ellas, pero las de fosfato salival fueron mayores en aquellos con caries. Pandey, et al. (17), Preethi, et al. (18), y Prabhakar, et al. (39), reportaron concentraciones menores de calcio en saliva sin estímulo en los pacientes con caries. Asimismo, en otros estudios en saliva total bajo estímulo se han reportado concentraciones menores de calcio y fosfato en los pacientes con caries $(40,45,46)$. En este estudio, las mayores concentraciones de calcio en la saliva de los niños con caries probablemente no se relacionan con las condiciones de secreción de la saliva, con estimulación o sin esta, aunque se han reportado correlaciones entre las concentraciones de calcio y las proteínas salivales (47).

Muchas de las proteínas salivales, como las mucinas, las estaterinas, las proteínas ricas en prolina y las histatinas (48), entre otras, experimentan un proceso de desnaturalización para formar parte de la película adquirida del esmalte, la cual penetra y se une a los cristales del esmalte para protegerlo de los ácidos (49-51). Durante este proceso de adsorción selectiva de las proteínas salivales sobre la superficie del esmalte, se rompen los enlaces con el calcio, lo cual favorece que más calcio libre se difunda fácilmente en la saliva y la biopelícula bacteriana (52); esto también podría explicar las concentraciones de calcio significativamente superiores en los dientes sanos del grupo ICDAS $>1$.

Las mayores concentraciones de fosfato en exudado gingival de dientes sanos en el grupo ICDAS>1 evidenciaron una asociación con las caries activas en las superficies de oclusión y con su gravedad, lo cual puede atribuirse a que los iones de fosfato que forman parte de la película adquirida del esmalte -la cual representa una barrera de permeabilidad selectiva para los iones con concentraciones de sobresaturación de calcio, fosfato y flúor con respecto a la apatita del esmalte-, disminuyen la difusión exterior y favorecen la remineralización del esmalte, lo cual reduce la desmineralización y la formación de caries. Por el contrario, las bajas concentraciones de saturación del fosfato en la película adquirida del esmalte se atribuyen a la pérdida de estos iones que reaccionan con los iones de hidrógeno producidos por el metabolismo microbiano de la biopelícula en presencia de carbohidratos fermentables, lo cual favorece la desmineralización y la pérdida de estructura de la hidroxiapatita $(53,54)$. 
Los resultados del presente trabajo y su análisis parecen indicar que la relación entre la bioquímica bucal y la caries dentaria constituye un sistema dinámico, complejo, multifactorial y de difícil modelación, lo cual explicaría la gran cantidad de resultados contrastantes de los diferentes trabajos publicados.

En conclusión, es muy factible que exista una asociación entre las variables estudiadas, la velocidad del flujo salivar y las concentraciones de calcio y de fosfato en la saliva y el líquido crevicular gingival, y la gravedad y actividad de la caries dentaria en la dentición primaria de niños escolares.

\section{Agradecimientos}

Al personal administrativo del área clínica del programa de la especialidad de Odontopediatría de la Facultad de Odontología de la Universidad de Carabobo y al personal administrativo de la Unidad de Investigaciones Morfopatológicas (UNIMPA), por su gentil y oportuna colaboración.

\section{Referencias}

1. Oropeza-Oropeza A, Molina-Frechero N, Castañeda-Castaneira E, Zaragoza-Rosado Y, Leyva DC. Caries dental en primeros molares permanentes de escolares de la delegación Tláhuac. Rev ADM. 2012;69:63-8.

2. Organización Mundial de la Salud. Salud bucodental. Nota informativa $N^{\circ} 318$. Abril de 2012. Fecha de consulta: 24 de agosto del 2017. Disponible en: http://www.who.int/ mediacentre/factsheets/fs318/es/

3. Petersen PE, Bourgeois D, Ogawa H, Estupiñán-Day S, Ndiaye C. The global burden of oral diseases and risks to oral health. Bull World Health Organ. 2005;83:661-9. https://doi. org/10.1590/S0042-96862005000900011

4. Villalobos-Rodelo JJ, Medina-Solís CE, Molina-Frechero N, Vallejos-Sánchez AA, Pontigo-Loyola AP, Espinoza-Beltrán JL. Caries dental en escolares de 6 a 12 años en Navolato, Sinaloa, México: experiencia, prevalencia, gravedad y necesidades de tratamiento. Biomédica. 2006;26:224-33. https://doi.org/10.7705/biomedica.v26i2.1412

5. Delgado-Angulo EK, Bernabé E. Influence of host related indicators on dental caries in the permanent dentition. Acta Odontol Latinoam. 2006;19:85-92.

6. Martins S, Álvarez E, Abanto J, Cabrera A, López R, Masoli C, et al. Epidemiología de la caries dental en America Latina. Relatório da mesa dos representantes das sociedades de Odontopediatria dos países Latinoamericanos. Rev Odontoped Latinoam. 2014;4:13-8.

7. Gomes PR, Costa SC, Cypriano S, Sousa ML. Situação da cárie dentária com relação às metas OMS 2000 e 2010. Cad Saúde Pública. 2004;20:866-70. https://doi.org/10.1590/ S0102-311X2004000300024

8. Morón A. Perfil epidemiológico bucal de las etnias venezolanas. Primer reporte nacional. Ciencia Odont. 2008;5(Supl.):11-134.

9. Peña E, Zavarce E. Prevalencia de caries dental utilizando el sistema internacional ICDAS en pacientes que acuden a consulta pediátrica en dos instituciones de la ciudad de Valencia, Estado Carabobo, Venezuela. Acta Odontol Venez. 2016;54.

10. Zambrano O, Fong L, Rivera L, Calatayud E, Hernández J, Maldonado A, et al. Impacto de la caries de infancia temprana en la calidad de vida del niño zuliano y familia. ODOUS Científica. 2015;16:8-17.

11. Rojas-Sánchez F. Algunas consideraciones sobre caries dental, fluoruros, su metabolismo y mecanismos de acción. Acta Odontol Venez. 2008;46:509-16.

12. Núñez DP, García L. Bioquímica de la caries dental. Rev Haban Cienc Med. 2010;9:156-66.

13. Garizoain G, Plischuk M, Salceda SA, Errecalde AL. Análisis de caries en superficies de la corona dentaria: estudio en una colección osteológica humana documentada. Rev Cienc Morfolog. 2017;19:1-8.

14. Vaillard-Jiménez E, Ortega A, Lezama G, Carrasco R, López C, Romano R. Características dimensionales de fosas y fisuras del esmalte de molares temporales. Rev Colomb Investig Odontol. 2012;3:114-23. 
15. Cortés A, Martignon S, Qvist V, Ekstrand KR. Approximal morphology as predictor of approximal caries in primary molar teeth. Clin Oral Investig. 2017;22:951-9. https://doi. org/10.1007/s00784-017-2174-3

16. Shimazaki Y, Fu B, Yonemoto K, Akifusa S, Shibata Y, Takeshita T, et al. Stimulated salivary flow rate and oral health status. J Oral Sci. 2017;59:55-62. https://doi.org/10.2334/josnusd.16-0372

17. Pandey P, Reddy NV, Rao VA, Saxena A, Chaudhary CP. Estimation of salivary flow rate, $\mathrm{pH}$, buffer capacity, calcium, total protein content and total antioxidant capacity in relation to dental caries severity, age and gender. Contemp Clin Dent. 2015;6:S65-71. https://doi. org/10.4103\%2F0976-237X.152943

18. Preethi BP, Reshma $\mathbf{D}$, Anand $\mathbf{P}$. Evaluation of flow rate, $\mathrm{pH}$, buffering capacity, calcium, total proteins and total antioxidant capacity levels of saliva in caries free and caries active children: An in vivo study. Indian J Clin Biochem. 2010;25:425-8. https://doi. org/10.1007\%2Fs12291-010-0062-6

19. Kardeşler L, Biyikoğlu B, Çetinkalp Ş, Pitkala M, Sorsa T, Buduneli N. Crevicular fluid matrix metalloproteinase-8, -13 , and TIMP-1 levels in type 2 diabetics. Oral Dis. 2010;16:476-81. https://doi.org/10.1111/j.1601-0825.2010.01659.x

20. Feres AR, Ferreira A, de Oliveira B, Ferreira M. Clinical oral and salivary parameters of children with juvenile idiopathic arthritis. Oral Surg Oral Med Oral Pathol Oral Radiol. 2014;117:75-80. https://doi.org/10.1016/i.0000.2013.08.024

21. International Caries Detection and Assessment System Coordinating Committee. Guía ICCMSTM para clínicos y educadores. Fecha de consulta: 24 de agosto del 2017. Disponible en: https://www.icdas.org/uploads/ICCMS-Guide-in-Spanish Oct2 2015FINAL\%20VERSION.pdf

22. Zambrano OR, Oliveira JM, Rivera LR, Añez YC, Finol AM. Prácticas de cuidado bucal en infancia temprana. Su asociación con caries dental y mal oclusiones. Ciencia Odontol. 2013;10:24-35

23. International Caries Detection and Assessment System Coordinating Committee. Rationale and evidence for the International Caries Detection and Assessment System (ICDAS II). Reviewed September 2011 (unchanged from 2005). Fecha de consulta: 24 de agosto de 2017. Disponible en: https://www.icdas.org/uploads/Rationale\%20and\%20 Evidence\%20ICDAS\%20II\%20revised\%20re\%20people\%20only\%202013.pdf

24. Cerda J, Villarroel L. Evaluación de la concordancia inter-observador en investigación pediátrica: coeficiente de kappa. Rev Chil Pediatr. 2008;79:54-8. https://doi.org/10.4067/ S0370-41062008000100008

25. Díaz-Cárdenas S, González-Martínez F. Prevalencia de caries dental y factores familiares en niños escolares de Cartagena de Indias, Colombia. Rev Salud Pública. 2010;12:843-51. https://doi.org/10.1590/S0124-00642010000500014

26. Sánchez-Pérez L, Méndez-Ramírez I, Sáenz-Martínez LR, Irigoyen-Camacho E, Mancera-Velázquez N, Acosta-Gío E. Línea basal de factores de riesgo a caries en escolares. Bol Med Hosp Infant Mex. 2005;62:33-44.

27. Ekstrand KR, Bjøndal L. Structural analyses of plaque and caries in relation to the morphology of the groove-fossa system on erupting mandibular third molars. Caries Res. 1997;31:336-48. https://doi.org/10.1159/000262416

28. Pitts NB, Zero DT, Marsh PD, Ekstrand K, Weintraub JA, Ramos-Gómez F, et al. Dental caries. Nat Rev Dis Primers. 2017;25:17030. https://doi.org/10.1038/nrdp.2017.30

29. Ribeiro AA, Azcárate-Peril MA, Cadenas MB, Butz N, Paster BJ, Chen T, et al. The ora bacterial microbiome of occlusal surfaces in children and its association with diet and caries. PLoS One. 2017;12:e0180621. https://doi.org/10.1371\%2Fjournal.pone.0180621

30. Arangannal P, Mahadev SK, Jayaprakash J. Prevalence of dental caries among schoo children in Chennai, based on ICDAS II. J Clin Diagn Res. 2016;10:ZC09-12. https://doi. org/10.7860\%2FJCDR\%2F2016\%2F14731.7523

31. Piovesan C, Ardenghi TM, Guedes RS, Ekstrand KR, Braga MM, Mendes FM. Activity assessment has little impact on caries parameters reduction in epidemiological surveys with preschool children. Community Dent Oral Epidemiol. 2013;41:204-11. https://doi. org/10.1111/cdoe.12004

32. Guedes RS, Piovesan C, Ardenghi TM, Emmanuelli B, Braga MM, Ekstrand KR, et al. Validation of visual caries activity assessment: A 2-yr cohort study. J Dent Res. 2014:93(Suppl.):101S-7S. https://doi.org/10.1177\%2F0022034514531017 
33. Foglio-Bonda PL, Brilli K, Pattarino F, Foglio-Bonda A. Salivary flow rate and $\mathrm{pH}$ in patients with oral pathologies. Eur Rev Med Pharmacol Sci. 2017;21:369-74.

34. Maeda EL, Sánchez RM, Verdugo RJ, Sánchez RA, Searcy R, Llodra JC. Flujo y capacidad amortiguadora salival en dos grupos de sujetos de 6 a 11 años de edad con bajo y alto índice de dientes cariados, perdidos y obturados. Univ Odontol. 2010;29:77-82.

35. Dogra S, Bhayya D, Arora R, Singh D, Thakur D. Evaluation of physio-chemical properties of saliva and comparison of its relation with dental caries. J Indian Soc Pedod Prev Dent. 2013;31:221-4. https://doi.org/10.4103/0970-4388.121816

36. Sullivan A. Correlation between caries incidence and secretion rate/buffer capacity of stimulated whole saliva in 5-7-year-old children matched for lactobacillus count and gingival state. Swed Dent J. 1990;14:131-5.

37. Sakeenabi B, Hiremath SS. Dental caries experience and salivary Streptococcus mutans, lactobacilli scores, salivary flow rate, and salivary buffering capacity among 6-yearold Indian school children. J Int Soc Prev Community Dent. 2011;1:45-51. https://doi. org/10.4103\%2F2231-0762.97697

38. Kaur A, Kwatra KS, Kamboj P. Evaluation of non-microbial salivary caries activity parameters and salivary biochemical indicators in predicting dental caries. J Indian Soc Pedod Prev Dent. 2012;30:212-7. https://doi.org/10.4103/0970-4388.105013

39. Prabhakar AR, Dodawad R, Raju OS. Evaluation of flow rate, $\mathrm{pH}$, buffering capacity, calcium, total protein and total antioxidant levels of saliva in caries free and caries active children - An in vivo study. Int J Clin Pediatr Dent. 2009;2:9-12. https://doi. org/10.5005\%2Fjp-journals-10005-1034

40. Fiyaz M, Ramesh A, Ramalingam K, Thomas B, Shetty S, Prakash P. Association of salivary calcium, phosphate, $\mathrm{pH}$ and flow rate on oral health: A study on 90 subjects. $\mathrm{J}$ Indian Soc Periodontol. 2013;17:454-60. https://doi.org/10.4103\%2F0972-124X.118316

41. Animireddy D, Reddy VT, Vallala P, Kotha SB, Ankireddy S, Mohammad N. Evaluation of $\mathrm{pH}$, buffering capacity, viscosity and flow rate levels of saliva in caries-free, minimal caries and nursing caries children: An in vivo study. Contemp Clin Dent. 2014;5:324-8. https://doi. org/10.4103\%2F0976-237X.137931

42. Lamanda A, Cheaib Z, Turgut MD, Lussi A. Protein buffering in model systems and in whole human saliva. PLoS One. 2007;2:e263. https://doi.org/10.1371\%2Fjournal. pone. 0000263

43. Jayaraj $\mathbf{D}$, Ganesan $\mathbf{S}$. Salivary $\mathrm{pH}$ and buffering capacity as risk markers for early childhood caries: A clinical study. Int J Clin Pediatr Dent. 2015;8:167-71. https://doi. org/10.5005\%2Fjp-journals-10005-1307

44. Kargül B, Yarat A, Tanboğa I, Emekli N. Salivary protein and some inorganic element levels in healthy children and their relationship to caries. J Marmara Univ Dent Fac. 1994;2:434-40.

45. Rajesh KS, Hegde S, Arun Kumar MS. Assessment of salivary calcium, phosphate, magnesium, $\mathrm{pH}$, and flow rate in healthy subjects, periodontitis, and dental caries. Contemp Clin Dent. 2015;6:461-5. https://doi.org/10.4103\%2F0976-237X.169846

46. Tulunoglu Ö, Demirtas S, Tulunoglu I. Total antioxidant levels of saliva in children related to caries, age, and gender. Int J Paediatr Dent. 2006;16:186-91. https://doi.org/10.1111/ j.1365-263X.2006.00733.x

47. Young JA, Schneyer CA. Composition of saliva in mammalia. Aust J Exp Biol Med Sci. 1981;59:1-53. https://doi.org/10.1038/icb.1981.1

48. Lee YH, Zimmermann JN, Custodio W, Xiao Y, Basiri T, Hatibovic-Kofmann S, et al. Proteomic evaluation of acquired enamel pellicle during in vivo formation. PLoS One. 2013;8:e67919. https://doi.org/10.1371\%2Fjournal.pone.0067919

49. Martins C, Castro GF, Siqueira MF, Xiao Y, Yamaguti PM, Siqueira WL. Effect of dialyzed saliva on human enamel demineralization. Caries Res. 2013;47:56-62. https://doi. org/10.1159/000343574

50. Hegde MN, Sajnani AR. Salivary proteins-A barrier on enamel demineralization: An in vitro study. Int J Clin Pediatr Dent. 2017;10:10-3. https://doi.org/10.5005\%2Fjpjournals-10005-1398

51. Hannig M, Hannig C. The pellicle and erosion. Erosive tooth wear. Monogr Oral Sci. 2014;25:206-14. https://doi.org/10.1159/000360376 
52. Ash A, Ridout MJ, Parker R, Machie AR, Burnett GR, Wilde PJ. Effect of calcium ions on in vitro pellicle formation from parotid and whole saliva. Colloids Surf B Biointerfaces. 2013;102:546-53. https://doi.org/10.1016/j.colsurfb.2012.08.048

53. González-Cabezas $\mathbf{C}$. The chemistry of caries: remineralization and demineralization events with direct clinical relevance. Dent Clin North Am. 2010;54:469-78. https://doi. org/10.1016/i.colen.2010.03.004

54. Hannig M, Joiner A. The structure, function and properties of the acquired pellicle. The teeth and their environment. Monogr Oral Sci. 2006;19:29-64. https://doi. org/10.1159/000090585 